\title{
Reviewing Michelson Interferometer Experiment and Measuring the Speed of Starlight
}

\author{
Jingshown $W^{1}{ }^{*}$, Hen-Wai Tsao ${ }^{1}$, Yen-Ru Huang ${ }^{2}$ \\ ${ }^{1}$ Department of Electrical Engineering, National Taiwan University, Taipei, Taiwan \\ ${ }^{2}$ Materials and Electro-Optics Research Divisions, National Chung-Shan Institute of Science and Technology, Taoyuan, Taiwan \\ Email: *wujsh@ntu.edu.tw
}

How to cite this paper: $\mathrm{Wu}, \mathrm{J}$., Tsao, H.-W. and Huang, Y.-R. (2019) Reviewing Michelson Interferometer Experiment and Measuring the Speed of Starlight. Journal of Modern Physics, 10, 539-547. https://doi.org/10.4236/jmp.2019.105037

Received: March 22, 2019

Accepted: April 21, 2019

Published: April 24, 2019

Copyright () 2019 by author(s) and Scientific Research Publishing Inc. This work is licensed under the Creative Commons Attribution International License (CC BY 4.0).

http://creativecommons.org/licenses/by/4.0/

\begin{abstract}
The wave-aether model was proposed long time ago. We study Michelson interferometer experiment and find that its theoretical calculation erroneously neglected the aether drag effect. We take the drag effect into account and reanalyze the theoretical interference pattern shift. The result is null because the drag coefficient of aether is zero. Such that the wave-aether model fulfills all light propagation characteristics. We design and implement a system to measure the starlight speed by comparing to that from a local source. We observe that the arrival times are different. It implies the apparent speeds of starlights are not equal to $c$.
\end{abstract}

\section{Keywords}

Wave-Aether Model, Michelson Interferometer, Speed of Light

\section{Introduction}

The speed and the propagation model of light are interesting subjects. The speed of light was considered as a very important physical parameter which is used to estimate the distance, mass etceteras. A few propagation models have been proposed since the ancient days [1] [2] [3] [4]. Among them, the wave-aether model attracts most attention, because this model satisfies many light propagation characteristics such as polarization, refraction, diffraction, interference, stellar aberration, etceteras. However, this model involved the existence and drag effect of aether [1] [3] [4] [5] [6]. Michelson-Morley used an interferometer to test the aether wind and they obtained a null result of interference pattern shift [1] [3] [4] [5]. The theoretical calculation gave a significant interference shift value. 
Both are inconsistent, which was a major obstacle for the wave-aether model. Because the Michelson-Morley experiment is an important milestone in modern physics, it still attracts some attention recently [6] [7] [8].

Lorentz and others proposed a hypothesis that the length of a moving object along its motion direction contracted by the factor of $\left(1-v^{2} / c^{2}\right)^{\frac{1}{2}}$, then the original Michelson's calculation interference pattern shift value agreed with the measured data [1]. In 1932, Kennedy and Thorndike performed an experiment using different arm lengths of an interferometer to test the Lorentz-Fizeau contraction [9]. The result showed the contraction might exist. Most people therefore gradually forgot and even abandoned this model.

In 1818, Fresnel predicted that the light would be dragged by the luminiferous medium [1] [2]. In 1725, Bradley discovered the stellar aberration. Fizeau and others used an apparatus to confirm the drag effect and the drag coefficient [1]. Now let us review Michelson's theoretical interference pattern shift calculation. We find that in his calculation, the drag effect was neglected, which yielded a significant shift value. When we take the drag effect into account to reanalyze the theoretical pattern shift value, we obtain a null result. So the measured data and the theoretical value are consistent. Thus we remove the major obstacle of the wave-aether model which now satisfies all light propagation characteristics. We may say that the wave-aether model is well-suited for describing the light propagation.

According to the wave-aether model, the motion of a light source will not communicate to the light speed i.e. the velocity of the light source is irrelevant to the speed of light but it will change the spectrum and wavelength.

Many observations and experiments have used moving light sources to test or prove the constancy of the speed of light.

In 1913, W. de Sitter observed some binary stars [10]. He concluded that the speed of light is independent of the speed of the light source and therefore the speed of light is constant. The wave-aether model explains his first conclusion. However, he did not consider the motion of the earth. The constancy of the speed of light conclusion conflicts with the wave-aether model and further evaluation is needed.

Around spring equinox each year, the earth is leaving away from Capella, Aldebaran, and Betelgeuse but approaching to Vega. We assume that the earth is moving in the aether, the starlight propagates with speed $c$ in the aether. We setup a system including a transmitter and a receiver. The transmitter simultaneously modulates starlight and light from a local source into pulses. These pulses are detected by a distant receiver. We compare the arrival times of these two kinds of pulses. However, the receiver has relative motion with respect to the aether, the apparent speed of starlight may be related to the motion of the earth according to the wave-aether model. We find the arrival times are different. The system setup and measurement will be described in Section 3. 


\section{Michelson-Morley Experiment and Aether Wind}

Michelson invented the interferometer to test the aether wind. Figure 1 shows the sketch of Michelson interferometer [1].

The two arms of Michelson interferometer are perpendicular. The length of the first arm is $l_{1}$ and a second one is $l_{2}$. The light from the source is incident upon the beam splitter B which splits the light into two parts. Assume that the thickness of the beam splitter is zero. One part of light travels along the first arm and is reflected back by the mirror M1. Then it travels along the same path and partially reflected by the beam splitter B incident upon the observer. According to Michelson calculation, the round trip time is

$$
t_{1}=\frac{l_{1}}{c-v}+\frac{l_{1}}{c+v}=\frac{2 l_{1}}{c^{2}-v^{2}}
$$

where $v$ is velocity of the aether wind [1].

Similarly the round trip time along the second arm is

$$
t_{2}=\frac{2 l_{2}}{\left(c^{2}-v^{2}\right)^{\frac{1}{2}}}
$$

Note that in the nineteenth century, the fluid mechanics was well developed and understood. The velocity of wave propagating in a fluid current, e.g. flowing water in a river, is vector addition. Probably, Michelson adopted the concept of fluid mechanics to formulate the light wave travelling times expressed as (1) and (2) which had neither theoretical basis nor experimental evidence.

The time difference of $t_{1}$ and $t_{2}$ is

$$
\Delta=t_{1}-t_{2} \approx \frac{2\left(l_{1}-l_{2}\right)}{c}+\frac{2 l_{1} v^{2}}{c^{3}}-\frac{l_{2} v^{2}}{c^{3}}
$$

As shown in Figure 1, the aether wind blows along $l_{1}$, we turn the interferometer $90^{\circ}$, the aether wind will blow along $I_{2}$, such that the round trip times along $l_{1}$ and $l_{2}$, i.e. $t_{1}$ and $t_{1}$, will be reversed and become $t_{1}{ }^{\prime}$ and $t_{2}{ }^{\prime}$. Now the time difference is

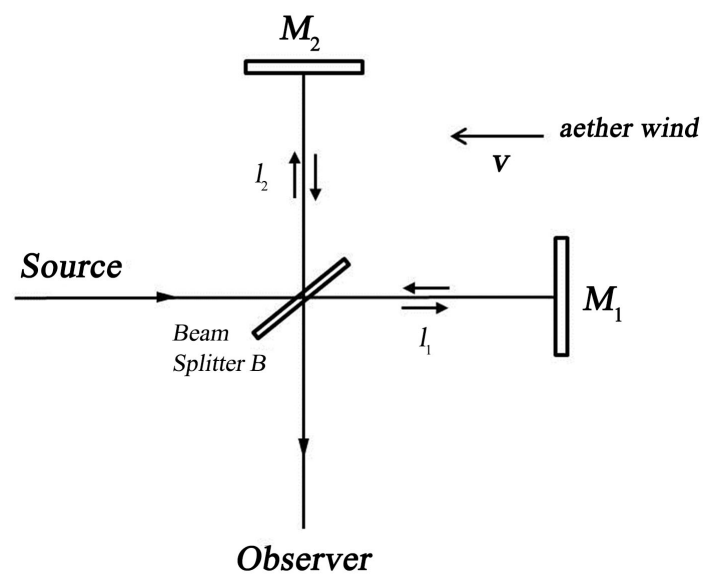

Figure 1. Sketch of Michelson interferometer. 


$$
\Delta^{\prime}=t_{1}{ }^{\prime}-t_{2}{ }^{\prime} \approx \frac{2\left(l_{1}-l_{2}\right)}{c}+\frac{l_{1} v^{2}}{c^{3}}-\frac{2 l_{2} v^{2}}{c^{3}}
$$

The difference $\Delta-\Delta^{\prime}$ would yield an interference pattern shift by $\delta$ fringes

$$
\delta=\frac{c\left(\Delta-\Delta^{\prime}\right)}{\lambda}=\frac{\left(l_{1}-l_{2}\right) v^{2}}{\lambda c^{2}}
$$

If $l_{1}=l_{2}$, then

$$
\delta=\frac{2(v / c)^{2}}{\lambda / l}
$$

where $\lambda$ is the wavelength, $v$ is the orbit speed of the earth [1].

Michelson used light with $\lambda=6 \times 10^{-7} \mathrm{~m}, l=1.2 \mathrm{~m}$.

Then according to (6) [1]:

$\delta=0.04$ fringes.

However, Michelson didn't observe this interference pattern shift. Later Michelson and Morley extended the arm length to $11.0 \mathrm{~m}$ to have more accurate result. They obtained 0.01 fringes. The theoretical interference shift is 0.40 fringes i.e. the difference of the theoretical value and the experimental data is ever bigger [1]. In about next three decades, many physicists used various arm lengths and still observed no significant interference pattern shift [1] [3] [4]. In those days, many physicists including Lorentz, etceteras believed that the aether might exist and the earth truly moved in the aether [1]. The mother nature had a magical power thus that the length of a moving object along its moving direction with respect to the aether contracted by a factor of $\left(1-v^{2} / c^{2}\right)^{\frac{1}{2}} \quad[1]$.

Then (1) became

$$
t_{1}=\frac{2 l_{1}}{\left(c^{2}-v^{2}\right)^{\frac{1}{2}}}
$$

and $t_{2}$ remained unchange as

$$
t_{2}=\frac{2 l_{2}}{\left(c^{2}-v^{2}\right)^{\frac{1}{2}}}
$$

Therefore

$$
\Delta=\Delta^{\prime}
$$

The theoretical value agreed with the experimental data.

In 1727, Bradley discovered the stellar aberration of $\gamma$-Draconis [11]. In 1871, Airy used a telescope in which the whole tube was filled with water, to observe the stellar aberration then he found the light drag effect, and gave the drag coefficient as [1] [11]

$$
f=1-\frac{1}{n^{2}}
$$

which is known as Fresnel's drag coefficient, $n$ is the refractive index.

Fizeau designed the apparatus as shown in Figure 2 to affirm the drag coeffi- 
cient as expressed in (10) [1]. For air, $n=1.0003$, the drag coefficient is 0.0006 . Imagine that if we fill the tubes of Fizeau's apparatus by a luminiferous medium with unit refractive index, such as aether, then $f=0$.

Based on previous discussion, readers may be aware of that using interferometer to test aether wind will yield a null result. Bearing the drag effect in mind i.e. $n=1, f=0$, we rewrite (1) and (2) as

$$
t_{1}=\frac{l_{1}}{\frac{c}{n}-v f}+\frac{l_{1}}{\frac{c}{n}+v f}=\frac{2 l_{1}}{c}
$$

and

$$
t_{2}=\frac{l_{1}}{\left(\frac{c^{2}}{n^{2}}-v^{2} f^{2}\right)^{1 / 2}}=\frac{2 l_{2}}{c}
$$

(11) and (12) show that the round trip times of lights travelling along the two arms are irrelevant to the velocity of aether wind. The theoretical value and the experimental data are consistent. The wave-aether model therefore fulfills all light propagation characteristics such as interference, polarization, stellar aberration, especially the null result of Michelson interferometer experiment.

In other words, the wave-aether model should be valid. Based on the wave-aether model, we design a system and perform the experiment to measure the speed of starlight.

\section{Measuring the Speed of Starlights}

In the wave-aether model, the speed of light is irrelevant to the speed of the source but is influenced by the motion of the observer. The principle of our measurement system is simple. We use a chopper to modulate the continuous starlight ray and a local light ray into pulses. The speed of the local light, $c$, is known [12]. We compare the arrival times of the starlight pulses and the local light pulses at the receiver. Then we can obtain the speeds of the starlights.

Figure 3 shows the latter version of this experimental setup. At the transmitter, for the main optical path, we use the Lulin One-meter Telescope (LOT) to collect the starlight. The first end of a multimode optical fiber is placed at the focal point of LOT and the other end is firmly fixed at the focal point of the off-axis parabolic mirror P1. The collimating ray is incident on the rotating mirror, M1, and then is incident on a second off-axis parabolic mirror P2. A 100 $\mu \mathrm{m}$ wide slit is put at the cofocal point of $\mathrm{P} 2$ and the third off-axis parabolic mirror P3 which makes the ray collimating again. When M1 spins, light pulses are generated after the slit. These pulses travel along the path P3, M2, M3, P4 and P5 then are incident on the photomultiplier tube (PMT) which converts the light pulses into electrical pulses. The end of the pigtail of an infaray light source is fixed at $\mathrm{T} 1$ to have a collimating ray which is merged into the main optical path by the beam splitter B1. After M1, the beam splitter B2 separates the infaray 
which is focalized at the end of another multimode fiber in T2. The multimode fiber plays as a slit and transmits the infaray pulses. At the receiver, the photodiode $(\mathrm{O} / \mathrm{E})$ converts the infaray pulses into electrical pulses as used for the reference and the trigger in the oscilloscope. The total distance from the transmitter to the receiver is $4302 \mathrm{~m}$. During the measurement, we alternately connect the first end of the multimode fiber to the LOT and the white light source to calibrate the system. We measured the arrival time of the light pulses from Capella, Aldebaran, Betelgense, and Vega in 2010, 2011, 2012, 2013 and 2014.

The details of the measurement setup, operation, data analysis, calibration, are described in References [13] [14] [15] [16].

Table 1 lists the delays of the starlight pulses respect to the white light pulses, which shows the arrival times of the starlight pulses different from the white light pulses. Note that negative delay of the starlight pulses implies that the speed of the starlight is faster than $c$ and positive delay indicates the speed of the starlight is slower than $c$. The trends of these results are roughly consistent with the wave-aether model qualitatively. Vega is approaching to the earth, so the negative delay with respect to the local white light was obtained. The delays were positive when the stars are leaving away from the earth, such as Capella, Betelgeuse, and Aldebaran. The orbital speed of the earth is about $30 \mathrm{~km} / \mathrm{s}$ on the ecliptic plane, so the delay is estimated about $1.4 \mathrm{~ns}$. The variation of measured delays may be caused by the system errors from temperature, humidity, and air dispersion [16], but the trends remain the same over the years.

Table 1. The delays of the starlight pulses with respect to the local white light pulses (unit: ns).

\begin{tabular}{ccccc}
\hline \multirow{2}{*}{ Date } & \multicolumn{3}{c}{ The delays of the starlight pulses (ns) } \\
\cline { 2 - 4 } & Capella & Betelgeuse & Aldebaran & Vega \\
\hline $2010 / 3 / 15$ & 1.61 & & & \\
$2010 / 3 / 16$ & $2.1 / 1.5$ & & -3.2 \\
$2010 / 3 / 18$ & 3.9 & 1.2 & -7.4 \\
$2011 / 2 / 28$ & $3.0 / 2.7$ & 1.8 & \\
$2011 / 3 / 4$ & 2.5 & & & \\
$2011 / 3 / 12$ & 3.8 & & & \\
$2012 / 2 / 18$ & 4.6 & & 2.1 & -1.3 \\
$2013 / 3 / 8$ & & & 2.2 & -1.9 \\
$2013 / 3 / 9$ & & & & \\
$2013 / 3 / 12$ & 1.2 & & 1.8 & \\
$2014 / 1 / 20$ & 1.4 & & 2.1 & \\
$2014 / 1 / 22$ & & & & \\
$2014 / 1 / 23$ & & & & \\
\hline
\end{tabular}




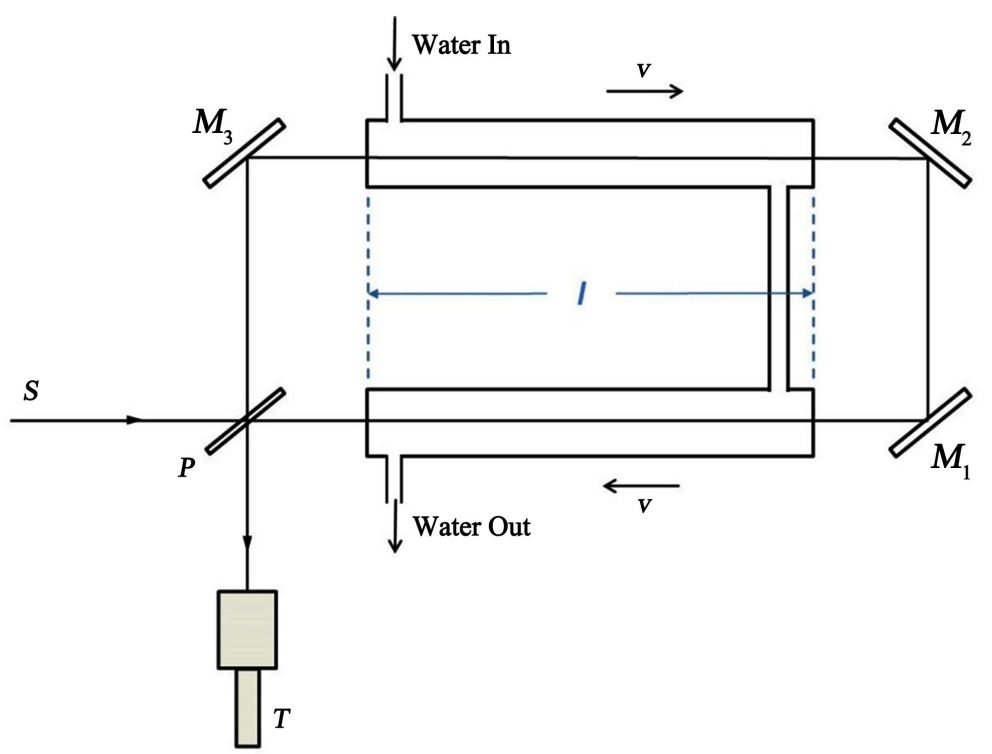

Figure 2. Sketch of Fizeau's drag apparatus.

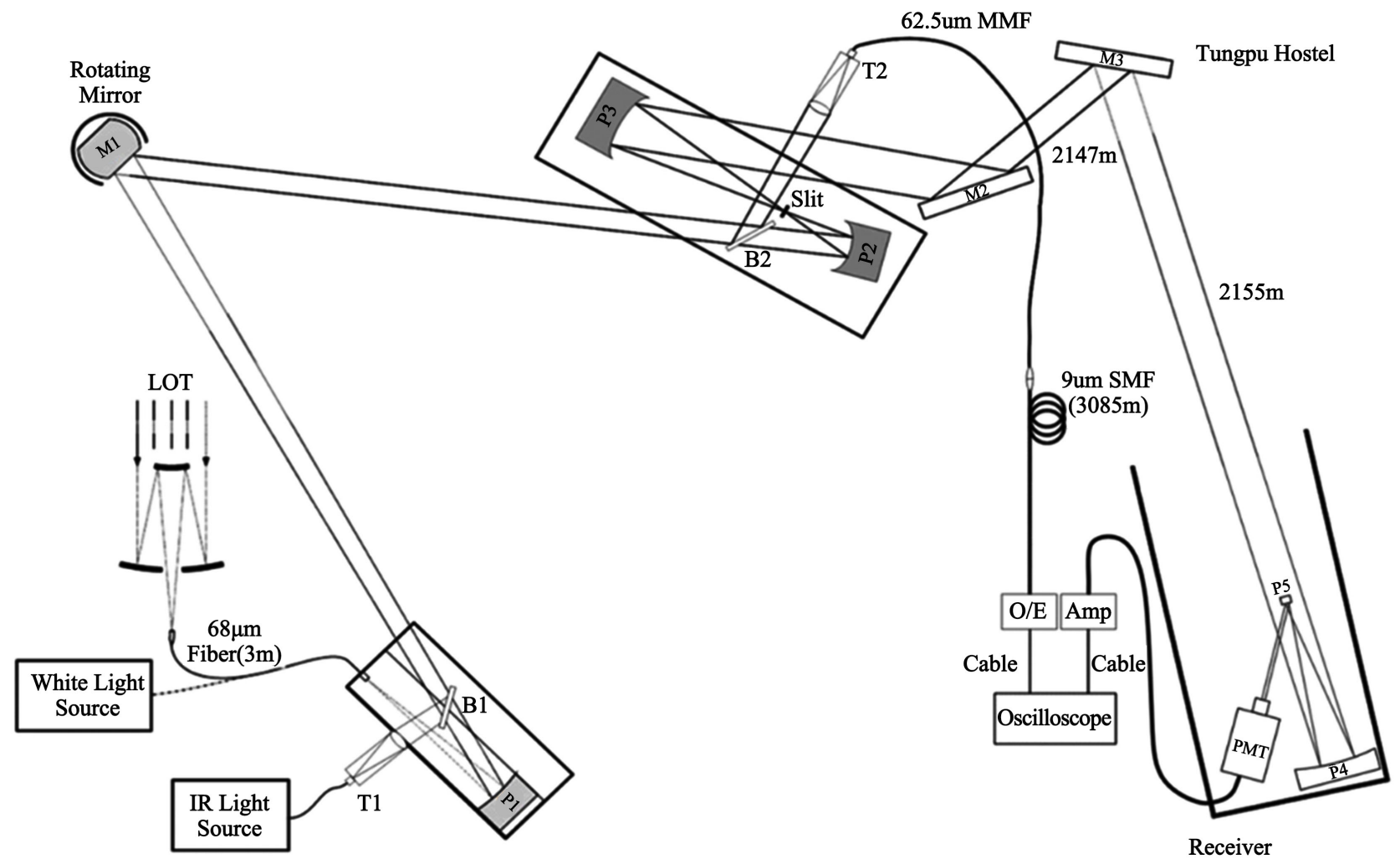

Figure 3. Sketch of the latter version of the experiment setup.

\section{Conclusions}

We study Michelson interferometer experiment and search the causes of disputation between the experimentally measured null interference pattern shift and the significant theoretical calculation value. We find that Michelson formulated the light travelling times along the two arms of the interferometer neglecting the 
drag effect. We take the drag coefficient into account to reanalyze the theoretical interference pattern shift value and obtain a null result. We prove that the measured data and the theoretical value match very well. Previously the disputation of Michelson interference pattern shift is a major concern of the validation of the wave-aether model for light propagation. Now this issue is solved and the wave-aether model satisfies all light propagation characteristics such as straight line transmission, polarization refraction, etceteras. We can conclude that the wave-aether model is valid.

Based on the wave-aether model, we consider that the earth moves in the aether. We setup a system on the earth to measure the speed of starlight i.e. the system moves in the stationary aether. Because the system including the observer is moving, the apparent measured speed of the starlight may not be equal to $c$. we design a transmitter to modulate the starlight and the light from a local source into pulses. Note that the speed of the local light pulses is $c$, these starlight and the local light pulses travel over a distance and reach the receiver. We compare the arrival times of these two kinds of pulses. We find that the arrival time of the starlight pulse is different with that of the local light pulse. It indicates that the apparent measured speeds of the starlight vary from the well known $c$. In general, our experiment fits the wave-aether model well qualitatively.

\section{Acknowledgements}

The authors are grateful for Prof. Hong T. Young, Prof. Wen-Ping Chen, Director Hung-Chin Lin, Prof. San-Liang Lee, Mr. Chong-Tsong Chang, the staff of the Lulin Observatory of National Central University and the Experimental Forest of National Taiwan University to provide the facilities and necessary help. We also thank assistance from Miss Hui-Ting Tsao, and many our assistants and students who were involved in this project in the last two decades.

\section{Supporting Information}

This work was supported in part by Excellent Research Projects of National Taiwan University and National Science Council, Taiwan under Grants 98R0062-06, NSC 97-2221-E-002-146-MY3, NSC100-2221-E-002-035, and NSC 101-2221-E-002-002.

\section{Conflicts of Interest}

The authors declare no conflicts of interest regarding the publication of this paper.

\section{References}

[1] French, A.P. (1968) Special Relativity. W. W. Norton \& Company Inc., New York.

[2] Huygens, C. (1690) Treatise on Light.

[3] Cassidy, D.C., Holton, G. and Rutherford, F.J. (2002) Understanding Physics, Birkhaiiser. 
[4] Panofsky, W.K.H. and Phillips, M. (1962) Classical Electricity and Magnetism. 2nd Edition, Addison-Wesley, Boston.

[5] Consoli, M., Matheson, C. and Pluchino, A. (2013) The European Physical Journal Plus, 128, 71. https://doi.org/10.1140/epjp/i2013-13071-7

[6] Consoli, M., Pluchino, A. and Rapisarda, A. (2016) EPL, 113, Article ID: 19001. https://doi.org/10.1209/0295-5075/113/19001

[7] Smid, T. (2017) European Journal of Physics, 38, Article ID: 065302. https://doi.org/10.1088/1361-6404/aa82f8

[8] Su, C.-C. (2001) EPL, 56, 468. https://doi.org/10.1209/epl/i2001-00502-1

[9] Kennedy, R.J. and Thorndike, E.M. (1932) Physical Review, 42, 400. https://doi.org/10.1103/PhysRev.42.400

[10] de Sitter, W. (1913) Proceedings of the Royal Netherlands Academy of Arts and Sciences, 15, 1297.

[11] Bradley, J. (1729) Philosophical Transactions of the Royal Society, 35, 637.

[12] Froome, K.D. and Essen, L. (1969) The Velocity of Light of Radio Waves. Academic Press, New York.

[13] Wu, J., Huang, Y.-R., Chang, S.-T., Tsao, H.-W., Lee, S.-L. and Lin, W.-C. (2016) Springer Proceedings in Physics, 177, 81.

[14] Wu, J., Chang, S.-T., Tsao, H.-W., Huang, Y.-R., Lee, S.-L., et al. (2013) Proceedings of SPIE, 8832, Article ID: 883203. https://doi.org/10.1117/12.2023127

[15] Huang, Y.-R., Wu, J., Chang, S.-T., Tsao, H.-W., Lee, S.-L. and Lin, W.-C. (2014) JPS Conference Proceedings, 1, Article ID: 014019.

[16] Huang, Y.-R. (2012) Measurement of Differences and Relativity between Speeds of Light from Various Stars. PhD Dissertation, National Taiwan University of Science and Technology. 